\title{
Spin-flip transitions and departure from the Rashba model in the Au(111) surface
}

\author{
Julen Ibañez-Azpiroz, ${ }^{1,2}$ Aitor Bergara, ${ }^{1,2,3}$ E. Ya. Sherman, ${ }^{4,5}$ and Asier Eiguren ${ }^{1,2}$ \\ ${ }^{1}$ Materia Kondentsatuaren Fisika Saila, Zientzia eta Teknologia Fakultatea, Euskal Herriko Unibertsitatea, \\ 644 Postakutxatila, 48080 Bilbao, Basque Country, Spain \\ ${ }^{2}$ Donostia International Physics Center (DIPC), Paseo Manuel de Lardizabal 4, 20018 Donostia/San Sebastian, Spain \\ ${ }^{3}$ Centro de Física de Materiales CFM - Materials Physics Center MPC, Centro Mixto CSIC-UPVIEHU, Edificio Korta, \\ Avenida de Tolosa 72, 20018 Donostia, Basque Country, Spain \\ ${ }^{4}$ Department of Physical Chemistry, University of Basque Country, UPV-EHU 48080 Bilbao, Bizkaia, Spain \\ ${ }^{5}$ IKERBASQUE, Basque Foundation for Science, Bilbao, Spain
}

(Received 28 June 2013; revised manuscript received 31 July 2013; published 3 September 2013; corrected 4 September 2013)

\begin{abstract}
We present a detailed analysis of the spin-flip excitations induced by a periodic time-dependent electric field in the Rashba prototype $\mathrm{Au}(111)$ noble metal surface. Our calculations incorporate the full spinor structure of the spin-split surface states and employ a Wannier-based scheme for the spin-flip matrix elements. We find that the spin-flip excitations associated with the surface states exhibit an strong dependence on the electron momentum magnitude, a feature that is absent in the standard Rashba model [E. I. Rashba, Sov. Phys. Solid State 2, 1109 (1960)]. Furthermore, we demonstrate that the maximum of the calculated spin-flip absorption rate is about twice the model prediction. These results show that, although the Rashba model accurately describes the spectrum and spin polarization, it does not fully account for the dynamical properties of the surface states.
\end{abstract}

DOI: 10.1103/PhysRevB.88.125404

PACS number(s): 71.70.-d, 72.25.Rb, 73.21.-b

\section{INTRODUCTION}

Surfaces represent an ideal testing ground for investigating the nature of the relativistic spin-orbit interaction in low dimensional systems. ${ }^{1-3}$ As pointed out in the pioneering work by LaShell et al., ${ }^{4}$ the lack of inversion symmetry of surfaces allows for the spin splitting of the Shockley-type surface states via the spin-orbit interaction. Noteworthy, the order of magnitude of the energy spin splitting is as much as $\sim 0.1-0.5 \mathrm{eV}$, about two orders of magnitude larger than in semiconductors. ${ }^{1}$ Recently, several studies performed on coated surfaces have revealed exceptional effects of the spinorbit interaction. The family of bismuth alloy surfaces, , $^{2,6}$ for instance, exhibits giant spin-orbit energy shifts of nearly $400 \mathrm{meV}$. Other interesting examples include the semiconducting surfaces $\mathrm{Tl} / \mathrm{Si}(111)-(1 \times 1),{ }^{7-9} \mathrm{Tl} / \mathrm{Ge}(111)-(1 \times 1),{ }^{10}$ and $\mathrm{Pb} / \mathrm{Ge}(111)-\beta \sqrt{3} \times \sqrt{3} R 30^{\circ},{ }^{11,12}$ among many others. In these systems, the bulk bands present a gap near the Fermi level, and thus the electron transport properties are strongly influenced by the spin-split metallic surface states. Even surfaces with light element overlayers such as $\mathrm{H} / \mathrm{W}(110)-(1 \times 1)$ reveal extremely complex spin polarization structure, which is inherent of the anisotropy of the spin-orbit interaction. ${ }^{13,14}$

A particularly appealing aspect about surfaces is the possibility of manipulating the electron spin by means of externally applied electric fields. ${ }^{15-20}$ The basic idea in this scenario would be to control the spin orientation by inducing spin-flip excitations between the spin-split surface states. In practice, this is done by applying an external electric field which couples to the spin-dependent electron velocity due to the spin-orbit interaction. Since electric fields are easily created and manipulated experimentally, the mentioned mechanism (electric dipole spin resonance) could offer new perspectives for future applications in spintronics.

In this paper, we present fully relativistic first-principles calculations for analyzing the spin-flip excitations induced by a time-dependent electric field in the Au(111) noble metal surface. This system is considered as the paradigm of a two-dimensional free-electron-like gas with the Rashba-type spin-orbit coupling., ${ }^{4,21}$ It is commonly accepted that the properties of the $\mathrm{Au}(111)$ surface states, such as the energy spin splitting or the spin polarization structure, are well described in terms of the Rashba model. ${ }^{1,22-25}$ In fact, the noble metal (111) surfaces have been considered as an almost perfect realization of the Rashba Hamiltonian. However, we demonstrate in this work that the spin related response properties of the $\mathrm{Au}(111)$ surface states show a detectable departure from this model. In particular, our calculations demonstrate that the spin-flip transition probability reveals an appreciable angular and momentum dependence, while in the Rashba model this quantity appears with a trivial functional shape. Furthermore, we find that the maximum value of the calculated spin-flip absorption rate is almost double the model prediction.

\section{THEORETICAL FRAMEWORK}

In this section, we briefly introduce the computational approach for analyzing the spin-flip excitations associated with the spin-split surface states. Unless otherwise stated, atomic units will be used throughout the work, $e=\hbar=m_{e}=4 \pi \epsilon_{0}=$ 1. Let us start by considering the following single-particle Hamiltonian including the spin-orbit interaction,

$$
\hat{\mathrm{H}}_{0}=\frac{\hat{\mathbf{p}}^{2}}{2}-V(\hat{\mathbf{r}})-\frac{1}{4 c^{2}} \hat{\boldsymbol{\sigma}} \cdot(\hat{\nabla} V(\hat{\mathbf{r}}) \times \hat{\mathbf{p}}),
$$

where $V(\hat{\mathbf{r}})$ is the scalar potential and $\hat{\boldsymbol{\sigma}}$ represent the Pauli spin operator. In this framework, we analyze the response of an electron state to an external time-dependent electric field described by the vector potential $\mathbf{A}_{\mathrm{ext}}(t)=\mathbf{A}_{0}^{(\alpha)} \cos \omega t$, where $\omega$ and $\alpha$ represent the frequency and polarization of the external field, respectively. The spatial variation of the field is neglected since we are interested in the optical limit $(\mathbf{q} \rightarrow 0)$, in which case the field can be safely considered as spatially constant. ${ }^{12,26}$ 
We adopt the following conventions for the $x$ and $y$ linearly polarized, $\mathbf{A}_{0}^{(x)}=A_{0} \hat{\mathbf{x}}$ and $\mathbf{A}_{0}^{(y)}=A_{0} \hat{\mathbf{y}}$, and the right $(R)$ and left $(L)$ circularly polarized light, $\mathbf{A}_{0}^{(R, L)}=A_{0}(\hat{\mathbf{x}} \pm i \hat{\mathbf{y}}) / \sqrt{2}$ [see Fig. 2(a) for the axes convention].

In our perturbation theory treatment, the leading term describing the interaction between the external field and the electron gas appears as ${ }^{16}$

$$
\hat{\mathrm{H}}_{\mathrm{int}}(t)=-\frac{1}{c} \hat{\mathbf{v}} \cdot \mathbf{A}_{\mathrm{ext}}(t),
$$

where $\hat{\mathbf{v}}$ represents the electron velocity operator, commonly expressed as

$$
\hat{\mathbf{v}}=-i\left[\hat{\mathbf{r}}, \hat{\mathrm{H}}_{0}\right]=\frac{\partial \hat{\mathrm{H}}_{0}}{\partial \hat{\mathbf{p}}}=\hat{\mathbf{p}}-\frac{1}{4 c^{2}}(\hat{\boldsymbol{\sigma}} \times \hat{\nabla} V(\hat{\mathbf{r}})) .
$$

We observe that apart from the canonical contribution $\hat{\mathbf{p}}$, the velocity operator contains an additional term which directly depends on the spin. It is precisely due to this term in Eq. (3) that the interaction term in Eq. (2) is allowed to produce spinflip transitions among spin-split surface states.

The transition rate associated to $\hat{\mathrm{H}}_{\mathrm{int}}(t)$ is calculated considering the ordinary first order perturbation (Fermi's golden rule),

$$
\begin{aligned}
\gamma_{m n}^{(\alpha)}(\omega)= & 2 \pi \int\left(f_{\mathbf{k} m}-f_{\mathbf{k} n}\right)\left|C_{m n}^{(\alpha)}(\mathbf{k})\right|^{2} \\
& \times \delta\left(\epsilon_{\mathbf{k} n}-\epsilon_{\mathbf{k} m}-\omega\right) \frac{d^{2} k}{(2 \pi)^{2}} .
\end{aligned}
$$

The above describes transitions from state $m$ to $n$, with $f_{\mathbf{k} i}$ and $\epsilon_{\mathbf{k} i}$ the Fermi-Dirac distribution function and surface state eigenvalue, respectively, with $i=m, n$.

The velocity operator, which is the origin of the electron spin-flip transitions, enters the matrix elements in Eq. (4) as

$$
C_{m n}^{(\alpha)}(\mathbf{k})=-\frac{1}{2 c} \mathbf{A}_{0}^{(\alpha)} \cdot\left\langle\Psi_{\mathbf{k} m}|\hat{\mathbf{v}}| \Psi_{\mathbf{k} n}\right\rangle,
$$

where $\Psi_{\mathbf{k} i}(\mathbf{r})=e^{i \mathbf{k} \cdot \mathbf{r}} \cdot u_{\mathbf{k} i}(\mathbf{r})$ represents the single-particle Bloch spinor wave function of surface states.

Considering the Ehrenfest theorem for the velocity operator, $\hat{\mathbf{v}}=-i\left[\hat{\mathbf{r}}, \hat{\mathrm{H}}_{0}\right]$, and expanding the commutator $\left[\hat{\mathbf{r}}, \hat{\mathrm{H}}_{0}\right]$, the matrix elements of Eq. (5) can be cast into the following form: 27,28

$$
C_{m n}^{(\alpha)}(\mathbf{k})=-\frac{\epsilon_{\mathbf{k} n}-\epsilon_{\mathbf{k} m}}{2 c} \mathbf{A}_{0}^{(\alpha)} \cdot\left\langle u_{\mathbf{k} m}\left|\hat{\nabla}_{\mathbf{k}}\right| u_{\mathbf{k} n}\right\rangle .
$$

The $\left\langle u_{\mathbf{k} m}\left|\hat{\nabla}_{\mathbf{k}}\right| u_{\mathbf{k} n}\right\rangle$ matrix element is precisely the generalized (non-Abelian) Berry connection ${ }^{29}$ associated to the spin-split surface states.

It is noteworthy that the spin noncollinearity is the ultimate reason why the above term does not vanish, as briefly illustrated in the next lines. Let us begin by considering a system without spin-orbit coupling and subjected to a constant magnetic field along the $z$ axis. In these conditions, the spinor states would be collinearly polarized along the $z$ axis, i.e., we would have $u_{\mathbf{k} n}(\mathbf{r})=g_{\mathbf{k} n}(\mathbf{r})(1,0)^{\mathrm{T}}$ and $u_{\mathbf{k} m}(\mathbf{r})=g_{\mathbf{k} m}(\mathbf{r})(0,1)^{\mathrm{T}}$, where $\mathrm{T}$ stands for matrix transposition. Since the momentum operator $\hat{\nabla}_{\mathbf{k}}$ is diagonal in the spin basis, one deduces that the matrix elements entering Eq. (6) vanish identically. This is in complete contrast to the situation when a finite spin-orbit interaction is present. This interaction induces an explicit momentum dependence of the spinor wave function, $u_{\mathbf{k} i}(\mathbf{r})=\left(g_{\mathbf{k} i}^{+}(\mathbf{r}), g_{\mathbf{k} i}^{-}(\mathbf{r})\right)^{\mathrm{T}}$. In this case, it is obvious that the spinor $\hat{\nabla}_{\mathbf{k}} u_{\mathbf{k} n}(\mathbf{r})=\left(\hat{\nabla}_{\mathbf{k}} g_{\mathbf{k} n}^{+}(\mathbf{r}), \hat{\nabla}_{\mathbf{k}} g_{\mathbf{k} n}^{-}(\mathbf{r})\right)^{\mathrm{T}}$ does not generally describe a spin orientation parallel to the original spinor $u_{\mathbf{k} n}(\mathbf{r})$. Thus an appreciable magnitude of the $\left\langle u_{\mathbf{k} m}\left|\hat{\nabla}_{\mathbf{k}}\right| u_{\mathbf{k} n}\right\rangle$ matrix elements entering Eq. (6) is a direct consequence of the spin noncollinearity introduced by the spin-orbit interaction. The opposite is also true and one deduces that if the direction of the spin polarization experiences a significant variation in some $\mathbf{k}$-space region, the associated spin-flip matrix elements would accordingly be enhanced, as found in a previous analysis. ${ }^{12}$

The calculation of the momentum gradient in Eq. (6) presents a computational challenge because of the inherent phase indeterminacy carried by the spinor wave functions. ${ }^{27}$ As a consequence, simple finite difference formulas cannot be directly applied, and a gauge fixing procedure is needed. Recently, a new method to solve this problem has been presented $^{28,30}$ whereby the matrix elements are reexpressed in terms of the maximally localized Wannier functions. ${ }^{31}$ In this scheme, the maximal localization of the Wannier states amounts to obtain the smoothest possible variation of the spinor states within the Brillouin zone, hence the interpolation procedure is optimized. Following this approach, the $C_{m n}^{\alpha}(\mathbf{k})$ matrix elements entering Eq. (4) can be interpolated into a very fine $\mathbf{k}$ mesh with a negligible computational cost. ${ }^{28,30,32}$ In the present work, the spin-flip matrix elements and surface state eigenvalues entering the integral of Eq. (4) have been evaluated in a dense $1000 \times 1000 \mathbf{k}$-point grid. This has allowed one to consider a very fine Gaussian width of $4 \times 10^{-4} \mathrm{eV}$ for the integral.

The ground state electronic properties of the $\mathrm{Au}(111)$ surface have been calculated within the noncollinear LDA-DFT formalism, considering a plane wave basis as implemented in the QUANTUM ESPRESSO package. ${ }^{34}$ We used a plane wave cutoff corresponding to $E_{c}=55 \mathrm{Ry}$ and a $32 \times 32$ Monkhorst-Pack mesh ${ }^{35}$ for the self-consistent cycle. The spin-orbit interaction has been incorporated considering normconserving fully relativistic pseudopotentials for the $5 d$ and

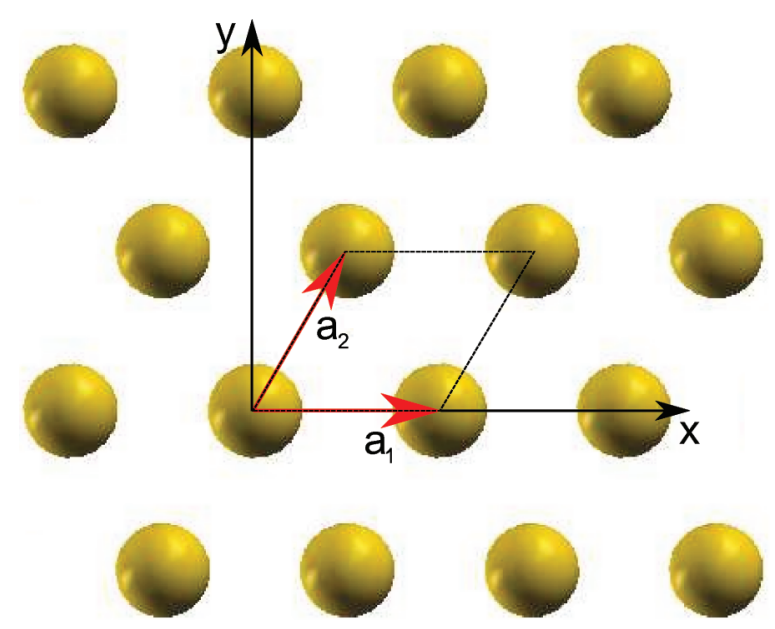

FIG. 1. (Color online) Top view of the Au(111) surface. ${ }^{33}$ Gold atoms are represented by the spheres (yellow). The big (red) arrows denote the $2 \mathrm{D}$ direct lattice defined in the text. 
$6 s$ electrons of $\mathrm{Au}$, including the full spinor structure of the wave functions. ${ }^{36}$ The $\mathrm{Au}(111)$ surface was modeled by the repeated slab technique considering 21 Au layers, with $21 \AA$ of vacuum separating the two sides of the slab and $\sim 2.49 \AA$ separating adjacent $\mathrm{Au}$ layers (interlayer spacing). In this surface, a single 2D lattice is described by the basis vector $\mathbf{a}_{1}=a \hat{\mathbf{x}}, \mathbf{a}_{2}=(\hat{\mathbf{x}}+\sqrt{3} \hat{\mathbf{y}}) a / 2$, with associated reciprocal basis vectors $\mathbf{b}_{1}=2 \pi / a(\hat{\mathbf{x}}-\hat{\mathbf{j}} / \sqrt{3}), \quad \mathbf{b}_{2}=2 \pi / a \cdot 2 \hat{\mathbf{y}} / \sqrt{3}$, where $a=3.12 \AA$. We have included the top view of the surface in Fig. 1. In our optimized configuration, all forces acting on individual atoms were smaller than $1.8 \times 10^{-4} \mathrm{Ry} \AA^{-1}$.

\section{RESULTS}

\section{A. Ground state properties}

In Fig. 2(a) we present the calculated electron band structure of the the $\mathrm{Au}(111)$ surface. The scalar relativistic (without spin-orbit interaction) and fully relativistic bands correspond to circles and solid lines, respectively. The bulk

(a)



(b)

(c)

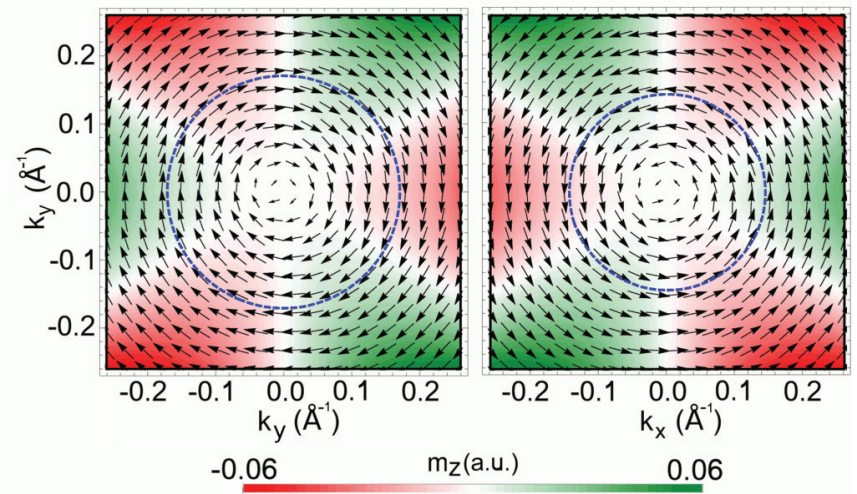

FIG. 2. (Color online) (a) Band structure of the Au(111) surface close to the $\bar{\Gamma}$ point $\left(\xi=0.5 \AA^{-1}\right)$. The scalar relativistic and fully relativistic bands are represented by circles and solid lines, respectively. The continuous background (gray) denotes the bulk band projection. (b), (c) Ab initio momentum dependent spin polarization associated to lower and higher spin-split subbands, respectively. Arrows represent the in-plane spin-polarization component, whereas the background color code indicates the surface perpendicular component, $m_{i, z}(\mathbf{k})$. Dashed (blue) lines indicate the calculated $a b$ initio Fermi surface associated with each surface subband. The radii of the circles in (b) and (c) are $k_{F}^{-}$and $k_{F}^{+}$, respectively. band projection is indicated by the background continuum (grey). While the scalar relativistic calculation shows a single spin-degenerate surface band outside the bulk band projection, the fully relativistic calculation shows the two well known spin-split metallic surface state bands measured by LaShell et al. ${ }^{4}$ We observe that, far from $\bar{\Gamma}$, the spin-split surface bands gradually spin degenerate as they approach the bulk projection (continuum) and become resonance states. The calculated binding energy at $\bar{\Gamma}$ is $420 \mathrm{meV}$, while the spin splitting at the Fermi level ranges approximately from 120 to $135 \mathrm{meV}$, corresponding to the Fermi wave vectors $k_{F}^{+}=0.145 \AA^{-1}$ and $k_{F}^{-}=0.175 \AA^{-1}$, respectively.

It is instructive to compare the calculated band structure of the surface states with the Rashba model energy dispersion of Eq. (A5). This model equation predicts a spin splitting that grows linearly with the magnitude of the electron momentum, $\Delta E=2|\mathbf{k}| \alpha_{R}$, with $\alpha_{R}$ the Rashba parameter. An explicit value for this parameter can be obtained by extracting $\Delta E$ and $|\mathbf{k}|$ from the $a b$ initio band structure. Since we are interested in the details close to the Fermi level, we consider the calculated Fermi wave vector, $|\mathbf{k}|=k_{F}^{+}=0.145 \AA^{-1}$, and the corresponding energy spin splitting, $\Delta E=0.12 \mathrm{eV}$, obtaining $\alpha_{R}=\Delta E / 2|\mathbf{k}|=0.419 \mathrm{eV} \AA$. This value is in good agreement with the one reported in a recent ARPES experiment, ${ }^{22}$ $\alpha_{R}=0.396 \mathrm{eV} \AA$. Additionally, we obtain an effective mass of $m^{*}=0.23$ [see Eq. (A5)] from a parabolic fit to the band structure, which also agrees well with experiments, ${ }^{22}$ $m^{*}=0.25$.

In Fig. 3 we compare the $a b$ initio band structure of the surface states with the Rashba model energy dispersion [Eq. (A5)] calculated using the parameter values $\alpha_{R}=0.419 \mathrm{eV} \AA$ and $m^{*}=0.23$. This figure shows a good agreement for energies below the Fermi level and, as expected, the ab initio and Rashba model energies coincide exactly at $|\mathbf{k}|=k_{F}^{+}$. For $|\mathbf{k}|>k_{F}^{+}$, the $a b$ initio energy spin splitting of the surface states ceases to grow linearly and, furthermore, it starts



FIG. 3. (Color online) Comparison between the Rashba model prediction for the energy dispersion [Eq. (A5)] and the calculated $a b$ initio band structure of the surface states. The Rashba parameter $\alpha_{R}$ has been fitted at $k_{F}^{+}=0.145 \AA^{-1}$, indicated by the vertical line. 


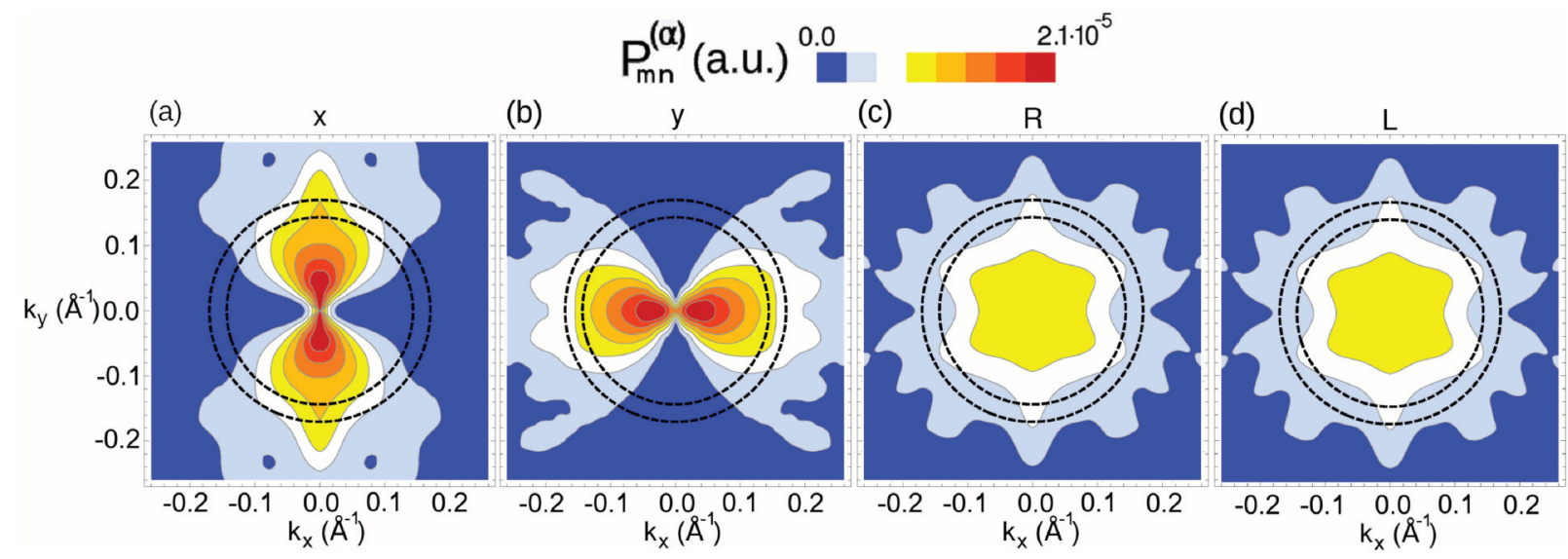

FIG. 4. (Color online) (a)-(d) Contour plot of the calculated momentum dependent surface state spin-flip transition probability, associated to the incoming light $\alpha=x, y, R$, and $L$ polarizations, respectively. The dashed lines (black) denote the $a b$ initio calculated Fermi surface associated with the surface states. The radii of the inner and outer circles are $k_{F}^{+}$and $k_{F}^{-}$, respectively.

decreasing. Therefore, the Rashba model energy dispersion clearly deviates from the ab initio band structure for $|\mathbf{k}|>k_{F}^{+}$.

Figures 2(b) and 2(c) illustrate the momentum dependent spin polarization of the spin-split surface states,

$$
\boldsymbol{m}_{i}(\mathbf{k})=\int \Psi_{\mathbf{k} i}^{*}(\mathbf{r}) \hat{\boldsymbol{\sigma}} \Psi_{\mathbf{k} i}(\mathbf{r}) d^{3} r
$$

In the figures, arrows represent the in-plane spin-polarization component, while the background code indicates the surface perpendicular component, $m_{z, i}(\mathbf{k})$.

Both surface states are spin polarized in practically the opposite direction in agreement with spin-resolved ARPES measurements, ${ }^{4,22}$ and describe a circular spin structure around the $\bar{\Gamma}$ point following the Rashba model [Eq. (A4)]. Our calculations confirm that $\boldsymbol{m}_{i}(\mathbf{k})$ is almost parallel to the surface for $|\mathbf{k}| \lesssim k_{F}^{+}$, which is the region where the Rashba model is expected to properly describe the properties of the surface states. Instead, the calculated surface-perpendicular component $\left[m_{z, i}(\mathbf{k})\right]$ acquires a finite value for $|\mathbf{k}| \gtrsim k_{F}^{+}$, indicating a departure from the Rashba model in this region. As shown by Henk et al. ${ }^{24}$ this feature is a consequence of in-plane components of the potential gradient associated with the real surface structure. In our calculations, we find that, at $k_{F}^{+}$, the surface-perpendicular component represents the $\sim 3 \%$ of the total magnitude of the spin polarization.

\section{B. Spin-flip transitions}

Figures 4(a), 4(b), 4(c), and 4(d) present the calculated momentum dependent spin-flip transition probability associated with the surface states,

$$
P_{m n}^{(\alpha)}(\mathbf{k}) \equiv \frac{\left|C_{m n}^{(\alpha)}(\mathbf{k})\right|^{2}}{\left|\mathbf{A}_{0}^{(\alpha)}\right|^{2}},
$$

for linearly $(\alpha=x, y)$ as well as for circularly $(\alpha=R, L)$ polarized light, respectively [see Eq. (5)].

In contrast to the Rashba model predicting a constant and totally isotropic transition probability for circularly polarized light [Eq. (A14)], our calculations presented in Figs. 4(c) and 4(d) describe an appreciable hexagonal angular dependence inherited from the $C_{3}$ symmetry of the real surface structure. For $x$ and $y$ linearly polarized light, our calculated spin-flip transition probability is in qualitative agreement with the dipolelike function predicted by the Rashba model [Eqs. (A12) and (A13)], but showing again an appreciable modulation.

Noteworthy, our ab initio calculations show a clear deviation from the Rashba model in one more important aspect: the dependence of the calculated spin-flip transition probability on the momentum magnitude $|\mathbf{k}|$. This feature is particularly evident for the $x$ and $y$ linearly polarized light [Figs. 4(a) and 4(b)], but it is also present for the $R$ and $L$ circular polarizations [Figs. 4(c) and 4(d)]. In all these cases, the spin-flip transition probability diminishes with increasing momentum, a feature that is absent in the ideal Rashba model. This can be understood as the surface bands approaching the bulk continuum lose gradually their surface character.

In Figs. 5(a) and 5(b) we explicitly analyze the angular dependence of the probability distribution for the $x$ and $y$ linearly polarized light, following several circular paths centered at high symmetry point $\bar{\Gamma}$ with fixed momentum $|\mathbf{k}|$. We observe that the calculated $P_{m n}^{(x)}(\mathbf{k})$ and $P_{m n}^{(y)}(\mathbf{k})$ closely follow the dipolelike functional shape of the Rashba model $\left(\sin ^{2} \varphi, \cos ^{2} \varphi\right.$ ), especially for small momenta, $|\mathbf{k}| \lesssim k_{F}^{+}$. We find that, even though the order of magnitude coincides for all $|\mathbf{k}|$, the calculated spin-flip transition probability shows a remarkable modulation with respect to the Rashba model result near $k_{F}^{+}$.

In Fig. 6 we present the calculated absorption rate associated to the spin-flip excitations,

$$
\Lambda_{m n}^{(\alpha)}(\omega)=\frac{\omega \cdot \gamma_{m n}^{(\alpha)}(\omega)}{\mathcal{P}},
$$

where $\gamma_{m n}^{(\alpha)}(\omega)$ is the spin-flip transition rate of Eq. (4), and $\mathcal{P}=\left|\mathbf{A}_{0}^{(\alpha)}\right|^{2} \omega^{2} / 8 \pi c$ is the optical power per unit area of the incident field. Thus $\Lambda_{m n}^{(\alpha)}(\omega)$ measures the percentage of the total irradiated light absorbed in the spin-flip processes.

We do not find any significant difference among the $x$ and $y$ linear polarization because of the isotropy of the problem, as the orbital components of the surface states in $\mathrm{Au}(111)$ are mainly $s, p_{z}$, and $d_{z^{2}}{ }^{22,23}$ The results for the $R$ and $L$ 

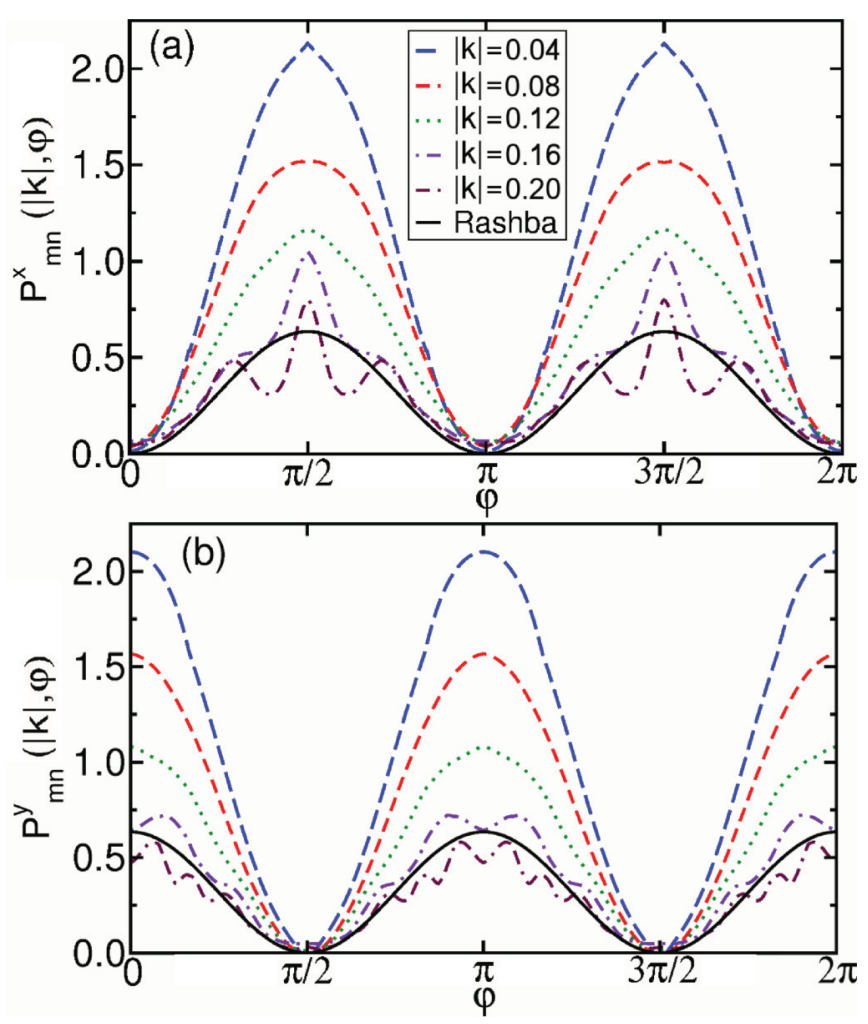

FIG. 5. (Color online) Calculated angular dependence of the spin-flip transition probability (units of $10^{-5}$ a.u.) for several circular paths corresponding to different absolute values of the electron momentum, $|\mathbf{k}|$ (units of $\AA^{-1}$ ). Panels (a) and (b) show the results for the incoming $x$ and $y$ linearly polarized light, respectively. The solid (black) lines are the $|\mathbf{k}|$-independent Rashba model predictions of Eqs. (A12) and (A13), with the parameter $\alpha_{R}$ extracted from the ab initio band structure at $k_{F}^{+}$.

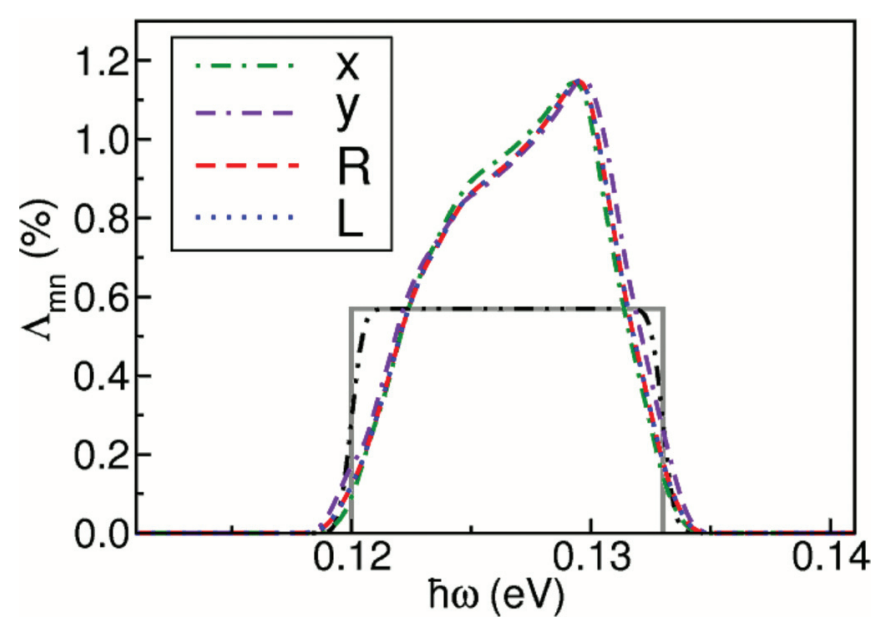

FIG. 6. (Color online) Integrated spin-flip absorption rate for the $\mathrm{Au}(111)$ surface. Superimposed dashed (red), dotted (blue), dot-dashed (green), and dot-dash-dashed (purple) lines represent the calculated results corresponding to the $x$ and $y$ linear and $R$ and $L$ circularly polarized light, respectively. The constant solid (gray) and dot-dot-dashed (black) lines denote respectively the Rashba model prediction without and with broadening. circular polarizations superimpose due to the presence of the time reversal symmetry. ${ }^{37,38}$ The solid (gray) line indicates the constant Rashba model prediction (for zero temperature), $\Lambda_{-+}=\pi / 4 c \sim 0.57 \%$, which is independent of the external field polarization and even of the Rashba parameter $\alpha_{R}$. We deduce from Fig. 6 that light is absorbed in the 120-135 $\mathrm{meV}$ energy window, corresponding to the range of the calculated energy spin splitting of the surface bands close to the Fermi level. Figure 6 reveals also that the Rashba model underestimates the maximum magnitude of the spin-flip absorption rate by approximately $100 \%$. The reason is that within the Brillouin zone area where spin-flip excitations are allowed $\left(k_{F}^{+}<|\mathbf{k}|<k_{F}^{-}\right.$; see Fig. 4), the calculated values for the transition probability matrix elements are also about twice the ones predicted by the Rashba model.

\section{CONCLUSIONS}

We have analyzed the spin-flip excitations induced by a time-dependent electric field in the Rashba model textbook example $\mathrm{Au}(111)$ surface. We have considered an $a b$ initio scheme based on maximally localized Wannier functions, including the full spinor structure of the surface states. Remarkably, the calculated ab-initio spin-flip transition probability exhibits an appreciable angular and momentum dependence, showing a much more complex structure than the Rashba model prediction. An important consequence of this modulation is that the maximum of the calculated spin-flip absorption rate is about twice the value predicted by the Rashba model. Thus, even though the Rashba model properly describes the ground state properties of the surface states, these results reveal that it does not fully account for the dynamical properties.

\section{ACKNOWLEDGMENTS}

We are grateful to Ivo Souza for very helpful discussions. The authors acknowledge financial support from UPV/EHU (Grant No. IT-366-07 and program UFI 11/55), the Spanish Mineco (Grants No. FIS2012-36673-C03-01 and No. FIS2009-12773-C02-01), and the Basque Government (Grant No. IT472-10), and thank the Donostia International Physics Center (DIPC) for providing the computer facilities.

\section{APPENDIX: SPIN-FLIP TRANSITIONS IN THE RASHBA MODEL}

In this Appendix we briefly describe the structure of the spin-flip transitions in the Rashba model for a two-dimensional (2D) free-electron-like gas, ${ }^{21}$ which is broadly considered as the standard model for analyzing the properties of surface states with spin-orbit interaction. ${ }^{1}$ Within this model, electrons are considered as free particles under the action of a simplified spin-orbit coupling,

$$
\hat{\mathrm{H}}_{\mathrm{R}}=\alpha_{R} \hat{\boldsymbol{\sigma}} \cdot(\hat{\mathbf{p}} \times \hat{\mathbf{z}}) .
$$

Above, $\alpha_{R}$ is the material-dependent Rashba parameter, $\hat{\mathbf{p}}=$ $-i \hat{\nabla}(\mathbf{r})$ is the electron momentum operator and $\hat{\mathbf{z}}$ is a unit vector pointing along the surface-perpendicular direction $z$. 
The expression of the Hamiltonian in the Rashba model is given by

$$
H_{0}=\frac{\hat{\mathbf{p}}^{2}}{2 m^{*}}+\alpha_{R} \hat{\boldsymbol{\sigma}} \cdot(\hat{\mathbf{p}} \times \hat{\mathbf{z}}),
$$

where $m^{*}$ is the electron effective mass. For an in-plane electron momentum $\mathbf{k}=k(\hat{\mathbf{x}} \cos \varphi+\hat{\mathbf{y}} \sin \varphi)$, the spinor eigenstates of the above Hamiltonian are given by ${ }^{1}$

$$
\Psi_{\mathbf{k}, \pm}(\mathbf{r})=\frac{e^{i \mathbf{k} \cdot \mathbf{r}}}{2 \pi} \frac{1}{\sqrt{2}}\left(\begin{array}{c}
i e^{-i \varphi / 2} \\
\pm e^{i \varphi / 2}
\end{array}\right) .
$$

Above, \pm denote the spin-up and spin-down subbands. The associated momentum dependent spin polarization is

$$
\mathbf{m}_{ \pm}(\mathbf{k})=\left\langle\Psi_{\mathbf{k}, \pm}|\boldsymbol{\sigma}| \Psi_{\mathbf{k}, \pm}\right\rangle=\frac{1}{2}(\hat{\mathbf{x}} \sin \varphi \mp \hat{\mathbf{y}} \cos \varphi),
$$

which is perpendicular to both the electron momentum $\mathbf{k}$ and the surface-perpendicular direction $\hat{\mathbf{z}}$.

The energy dispersion corresponding to the spinor states of Eq. (A3) is

$$
\epsilon_{k, \pm}=\frac{k^{2}}{2 m^{*}} \pm \alpha_{R} k
$$

Thus the eigenstates of the Rashba model Hamiltonian show an energy spin splitting, which increases linearly with the electron momentum magnitude,

$$
\Delta E_{k}=\epsilon_{k,+}-\epsilon_{k,-}=2 \alpha_{R} k .
$$

Due to the Rashba spin-orbit coupling term of Eq. (A1), the velocity operator, $\hat{\mathbf{v}}=\partial \hat{\mathrm{H}}_{0} / \partial \hat{\mathbf{p}}$ [Eq. (3)], becomes spin dependent,

$$
\begin{aligned}
& v_{x}=\frac{\hat{p}_{x}}{m^{*}}-\alpha_{R} \hat{\sigma}_{y}, \\
& v_{y}=\frac{\hat{p}_{y}}{m^{*}}+\alpha_{R} \hat{\sigma}_{x} .
\end{aligned}
$$

Considering the above expressions, the transition matrix elements between the $\Psi_{\mathbf{k}, \pm}(\mathbf{r})$ states [Eq. (5)],

$$
C_{-+}^{(\alpha)}(\mathbf{k})=-\frac{e}{2 c} \mathbf{A}_{0}^{(\alpha)} \cdot\left\langle\Psi_{-\mathbf{k}}|\hat{\mathbf{v}}| \Psi_{+\mathbf{k}}\right\rangle,
$$

are directly accessible.

First, it is worth noting that the spin-diagonal part of the velocity operator (the canonical contribution $\hat{\mathbf{p}} / \mathrm{m}^{*}$ ) does not contribute to $C_{-+}^{(\alpha)}(\mathbf{k})$ due to the orthogonality of the $\Psi_{ \pm \mathbf{k}}(\mathbf{r})$ states in spin basis. Therefore, the only finite contribution to $C_{-+}^{(\alpha)}(\mathbf{k})$ is proportional to the Pauli matrices appearing in Eq. (A7). We find the following expressions for different light polarizations:

$$
\begin{gathered}
C_{-+}^{(x)}(\varphi)=\frac{A_{0}}{2 c}\left\langle\Psi_{-\mathbf{k}}\left|\alpha_{R} \hat{\sigma}_{y}\right| \Psi_{+\mathbf{k}}\right\rangle=i \frac{\alpha_{R} A_{0}}{2 c} \sin \varphi, \\
C_{-+}^{(y)}(\varphi)=-\frac{A_{0}}{2 c}\left\langle\Psi_{-\mathbf{k}}\left|\alpha_{R} \hat{\sigma}_{x}\right| \Psi_{+\mathbf{k}}\right\rangle=-i \frac{\alpha_{R} A_{0}}{2 c} \cos \varphi, \\
C_{-+}^{(R, L)}(\varphi)=\frac{e A_{0}}{2 \sqrt{2} c}\left\langle\Psi_{-\mathbf{k}}\left|\alpha_{R} \hat{\sigma}_{y} \mp i \alpha_{R} \hat{\sigma}_{x}\right| \Psi_{+\mathbf{k}}\right\rangle \\
= \pm \frac{\alpha_{R} A_{0}}{2 \sqrt{2} c} e^{\mp i \varphi} .
\end{gathered}
$$

Therefore, the transition matrix elements depend only on the direction of the electron momentum $\varphi$, but not on the magnitude $k$. The associated spin-flip transition probability, $P_{-+}^{(\alpha)}(\mathbf{k}) \equiv\left|C_{-+}^{(\alpha)}(\mathbf{k})\right|^{2} /\left|\mathbf{A}_{0}^{(\alpha)}\right|^{2}$ [see Eq. (8)], is straightforwardly obtained from the above expressions,

$$
\begin{gathered}
P_{-+}^{(x)}(\varphi)=\frac{\alpha_{R}^{2}}{4 c^{2}} \sin ^{2} \varphi, \\
P_{-+}^{(y)}(\varphi)=\frac{\alpha_{R}^{2}}{4 c^{2}} \cos ^{2} \varphi . \\
P_{-+}^{(R)}=P_{-+}^{(L)}=\frac{\alpha_{R}^{2}}{8 c^{2}} .
\end{gathered}
$$

The last equation shows that the transition probability for $R$ and $L$ polarized light is identical and independent of the electron momentum.

Finally, we have all the elements needed to compute the spin-flip absorption rate [Eq. (4)],

$$
\begin{aligned}
\Lambda_{-+}^{(\alpha)}(\omega) & =\frac{\omega}{\mathcal{P}} 2 \pi \int \frac{d^{2} k}{(2 \pi)^{2}} F_{k}\left|C_{-+}^{(\alpha)}(\varphi)\right|^{2} \delta\left(\Delta E_{k}-\omega\right) \\
& =\frac{4}{c\left|A_{0}\right|^{2} \omega} G^{(\alpha)} \int d k F_{k} k \delta\left(2 \alpha_{R} k-\omega\right),
\end{aligned}
$$

where $F_{k}=f_{k,-}-f_{k,+}$ takes into account the Fermi occupation factors,

$$
f_{k, \pm}=\left[\exp \left(\frac{k^{2} / 2 m^{*} \pm \alpha_{R} k-E_{F}}{k_{B} T}\right)+1\right]^{-1},
$$

with $E_{F}$ the Fermi energy, $k_{B}$ the Boltzmann constant, and $T$ the temperature. In Eq. (A15), $G^{(\alpha)}$ denotes the integration of the angular part, which yields the same result for all light polarizations [see Eqs. (A9)-(A11)],

$$
G^{(\alpha)}=\int_{0}^{2 \pi} d \varphi\left|C_{-+}^{(\alpha)}(\varphi)\right|^{2}=\frac{\left|A_{0}\right|^{2} \pi \alpha_{R}^{2}}{4 c^{2}} .
$$

Inserting Eq. (A17) into Eq. (A15) and integrating over $k$, we finally obtain

$$
\Lambda_{-+}^{(\alpha)}(\omega)=\frac{\pi}{4 c}\left(f_{\omega,-}-f_{\omega,+}\right)
$$

The spin-flip absorption rate in the Rashba model, Eq. (A18), turns out to be independent of the external field polarization. Furthermore, it is almost independent of the Rashba parameter $\alpha_{R}$, which enters only through the occupation factors. Indeed, for $T \rightarrow 0$ we have that

$$
\lim _{T \rightarrow 0} \Lambda_{-+}^{(\alpha)}(\omega)= \begin{cases}\frac{\pi}{4 c} & \text { if } \omega^{2} / 2+\alpha_{R} \omega>E_{F} \\ & \text { and } \omega^{2} / 2-\alpha_{R} \omega<E_{F}, \\ 0 & \text { otherwise. }\end{cases}
$$

Therefore, we conclude that the parameter $\alpha_{R}$ does not affect the magnitude of the light absorption rate, but only the range of frequencies in which light is absorbed. 
${ }^{1}$ M. Heide, G. Bihlmayer, P. Mavropoulos, A. Bringer, and S. Blügel, Psi-k Newsletter 78, 1109 (2006).

${ }^{2}$ J. I. Pascual, G. Bihlmayer, Y. M. Koroteev, H.-P. Rust, G. Ceballos, M. Hansmann, K. Horn, E. V. Chulkov, S. Blügel, P. M. Echenique et al., Phys. Rev. Lett. 93, 196802 (2004).

${ }^{3}$ A. Strozecka, A. Eiguren, and J. I. Pascual, Phys. Rev. Lett. 107, 186805 (2011).

${ }^{4}$ S. LaShell, B. A. McDougall, and E. Jensen, Phys. Rev. Lett. 77, 3419 (1996).

${ }^{5}$ S. V. Eremeev, I. A. Nechaev, Y. M. Koroteev, P. M. Echenique, and E. V. Chulkov, Phys. Rev. Lett. 108, 246802 (2012).

${ }^{6}$ C. R. Ast, J. Henk, A. Ernst, L. Moreschini, M. C. Falub, D. Pacilé, P. Bruno, K. Kern, and M. Grioni, Phys. Rev. Lett. 98, 186807 (2007).

${ }^{7}$ K. Sakamoto, T. Oda, A. Kimura, K. Miyamoto, M. Tsujikawa, A. Imai, N. Ueno, H. Namatame, M. Taniguchi, P. E. J. Eriksson et al., Phys. Rev. Lett. 102, 096805 (2009).

${ }^{8}$ M.-H. Liu and C.-R. Chang, Phys. Rev. B 80, 241304 (2009).

${ }^{9}$ J. Ibañez-Azpiroz, A. Eiguren, and A. Bergara, Phys. Rev. B 84, 125435 (2011).

${ }^{10}$ Y. Ohtsubo, S. Hatta, H. Okuyama, and T. Aruga, J. Phys.: Condens. Matter 24, 092001 (2012).

${ }^{11}$ K. Yaji, Y. Ohtsubo, S. Hatta, H. Okuyama, K. Miyamoto, T. Okuda, A. Kimura, H. Namatame, M. Taniguchi, and T. Aruga, Nat. Commun. 1, 1 (2010).

${ }^{12}$ J. Ibañez-Azpiroz, A. Eiguren, E. Ya. Sherman, and A. Bergara, Phys. Rev. Lett. 109, 156401 (2012).

${ }^{13}$ M. Hochstrasser, J. G. Tobin, E. Rotenberg, and S. D. Kevan, Phys. Rev. Lett. 89, 216802 (2002).

${ }^{14}$ A. Eiguren and C. Ambrosch-Draxl, New J. Phys. 11, 013056 (2009).

${ }^{15}$ S. Datta and B. Das, Appl. Phys. Lett. 56 (1990).

${ }^{16}$ E. I. Rashba and A. L. Efros, Phys. Rev. Lett. 91, 126405 (2003).
${ }^{17}$ E. I. Rashba and V. Sheka, Sov. Phys. Solid State 3, 1357 (1961).

${ }^{18}$ V. N. Golovach, M. Borhani, and D. Loss, Phys. Rev. B 74, 165319 (2006).

${ }^{19}$ D. V. Khomitsky, L. V. Gulyaev, and E. Y. Sherman, Phys. Rev. B 85, 125312 (2012).

${ }^{20}$ E. I. Rashba, Phys. Rev. B 70, 201309 (2004).

${ }^{21}$ E. I. Rashba, Sov. Phys. Solid State 2, 1109 (1960).

${ }^{22}$ J. Henk, M. Hoesch, J. Osterwalder, A. Ernst, and P. Bruno, J. Phys.: Condens. Matter 16, 7581 (2004).

${ }^{23}$ M.-H. Liu, S.-H. Chen, and C.-R. Chang, Phys. Rev. B 78, 195413 (2008).

${ }^{24}$ J. Henk, A. Ernst, and P. Bruno, Phys. Rev. B 68, 165416 (2003).

${ }^{25}$ J. Premper, M. Trautmann, J. Henk, and P. Bruno, Phys. Rev. B 76, 073310 (2007).

${ }^{26}$ E. Y. Sherman, Phys. Rev. B 67, 161303 (2003).

${ }^{27}$ E. I. Blount, Solid State Phys. 13, 305 (1962).

${ }^{28}$ X. Wang, J. R. Yates, I. Souza, and D. Vanderbilt, Phys. Rev. B 74, 195118 (2006)

${ }^{29}$ M. V. Berry, Proc. R. Soc. A 392, 45 (1984).

${ }^{30}$ M. G. Lopez, D. Vanderbilt, T. Thonhauser, and I. Souza, Phys. Rev. B 85, 014435 (2012).

${ }^{31}$ A. A. Mostofi, J. R. Yates, Y. Lee, I. Souza, D. Vanderbilt, and N. Marzari, Comput. Phys. Commun. 178, 685 (2008).

${ }^{32}$ X. Wang, D. Vanderbilt, J. R. Yates, and I. Souza, Phys. Rev. B 76, 195109 (2007).

${ }^{33}$ A. Kokalj, Comput. Mater. Sci. 28, 155 (2003).

${ }^{34}$ P. Giannozzi, S. Baroni, N. Bonini, M. Calandra, R. Car, C. Cavazzoni, D. Ceresoli, G. L. Chiarotti, M. Cococcioni, I. Dabo, et al., J. Phys.: Condens. Matter 21, 395502 (2009).

${ }^{35}$ H. J. Monkhorst and J. D. Pack, Phys. Rev. B 13, 5188 (1976).

${ }^{36}$ A. Dal Corso and A. Mosca Conte, Phys. Rev. B 71, 115106 (2005).

${ }^{37}$ I. Souza and D. Vanderbilt, Phys. Rev. B 77, 054438 (2008).

${ }^{38}$ W. Yao, D. Xiao, and Q. Niu, Phys. Rev. B 77, 235406 (2008). 